Irish Math. Soc. Bulletin

Number 82, Winter 2018, 79-90

ISSN 0791-5578

\title{
Computing the Varchenko Determinant of a Bilinear Form
}

\author{
HERY RANDRIAMARO
}

\begin{abstract}
The Varchenko determinant is the determinant of the bilinear form associated to a real hyperplane arrangement. We show that we can obtain the exact value of this determinant for certain hyperplane arrangements if we know the edges which are relevant.
\end{abstract}

\section{INTRODUCTION}

Let $x=\left(x_{1}, \ldots, x_{n}\right)$ be a variable of the Euclidean space $\mathbb{R}^{n}$, and $a_{1}, \ldots, a_{n}, b$ real coefficients such that $\left(a_{1}, \ldots, a_{n}\right) \neq(0, \ldots, 0)$. A hyperplane $H$ of $\mathbb{R}^{n}$ is a $(n-1)$-dimensional affine subspace $H:=\left\{x \in \mathbb{R}^{n} \mid a_{1} x_{1}+\cdots+a_{n} x_{n}=b\right\}$. An arrangement of hyperplanes in $\mathbb{R}^{n}$ is a finite set of hyperplanes. The most famous hyperplane arrangement is certainly the braid arrangement $\mathcal{B}_{n}=\left\{\left\{x \in \mathbb{R}^{n} \mid x_{i}-x_{j}=0\right\}\right\}_{1 \leq i<j \leq n}$. Hyperplane arrangement theory is currently a very active area of research, combining ideas from algebraic combinatorics, algebraic topology, and algebraic geometry. In the preface of their book [3], Orlik and Terao wrote Arrangements are easily defined and may be enjoyed at levels ranging from the recreational to the expert, yet these simple objects lead to deep and beautiful results. Their study combines methods from many areas of mathematics and reveals unexpected connections. The bilinear form of a hyperplane arrangement defined by Varchenko confirms their affirmation. The Varchenko determinant is the determinant of this bilinear form.

An edge of a hyperplane arrangement $\mathcal{A}$ is a nonempty intersection of some of its hyperplanes. Denote by $L(\mathcal{A})$ the set of all edges of

2010 Mathematics Subject Classification. 05A05, 05A19.

Key words and phrases. Bilinear Form, Hyperplane Arrangement, Varchenko Determinant.

Received on 20-12-2017; revised 16-8-2018.

Support from International Centre for Theoretical Physics through Visiting Programme is gratefully acknowledged. 
$\mathcal{A}$. The arrangement of hyperplanes in $\mathcal{A}$ containing an edge $E$ in $L(\mathcal{A})$ is

$$
\mathcal{A}_{E}:=\{H \in \mathcal{A} \mid E \subseteq H\} .
$$

The hyperplane arrangement in the edge $E$ cut by $\mathcal{A}$ is

$$
\mathcal{A}^{E}:=\{H \cap E \mid H \in \mathcal{A}, E \nsubseteq H\} .
$$

A chamber of a hyperplane arrangement $\mathcal{A}$ is a connected component of the complement $\mathbb{R}^{n} \backslash \bigcup_{H \in \mathcal{A}} H$. Denote the set of all chambers of $\mathcal{A}$ by $\mathfrak{C}(\mathcal{A})$.

Assign a variable $a_{H}$ called weight to each hyperplane $H$ of an arrangement $\mathcal{A}$. Define the weight a $(E)$ of an edge $E$ by

$$
\mathrm{a}(E):=\prod_{\substack{H \in \mathcal{A} \\ E \subseteq H}} a_{H} .
$$

The multiplicity of an edge $E$ is

$$
l(E):=n(E) p(E),
$$

where $n(E):=\left|\mathfrak{C}\left(\mathcal{A}^{E}\right)\right|$, and $p(E)$ is defined below.

For every edge $E$ of codimension $r$, let $N$ be an $r$-dimensional normal subspace to $E$. All hyperplanes of the resulting arrangement $\left(\mathcal{A}_{E}\right)^{N}$ pass through the point $\{v\}=E \cap N$. Consider the hyperplane arrangement which $\left(\mathcal{A}_{E}\right)^{N}$ induces in the tangent space $T_{v} N$. It determines another hyperplane arrangement $P \mathcal{A}_{E}$ in the projectivization of $T_{v} N$.

A chamber of an arrangement is bounded with respect to a hyperplane if the closure of this chamber does not intersect the hyperplane. For any arrangement $\mathcal{A}^{\prime}$ in a real projective space, the numbers of chambers which are bounded with respect to its hyperplanes are all the same [5, Theorem 1.5], and we denote this number by $e\left(\mathcal{A}^{\prime}\right)$.

Finally, for an edge $E$ in $L(\mathcal{A})$, define $p(E):=e\left(P \mathcal{A}_{E}\right)$.

Let $R_{\mathcal{A}}=\mathbb{Z}\left[a_{H} \mid H \in \mathcal{A}\right]$ be the ring of polynomials in variables $a_{H}$. The module of $R_{\mathcal{A}}$-linear combinations of chambers of the hyperplane arrangement $\mathcal{A}$ is

$$
M_{\mathcal{A}}:=\left\{\sum_{C \in \mathfrak{C}(\mathcal{A})} x_{C} C \mid x_{C} \in R_{\mathcal{A}}\right\}
$$

Let $\mathcal{H}(C, D)$ be the set of hyperplanes separating the chambers $C$ and $D$ in $\mathfrak{C}(\mathcal{A})$. Define the $R_{\mathcal{A}}$-bilinear symmetric form 
$\mathrm{B}: M_{\mathcal{A}} \times M_{\mathcal{A}} \rightarrow R_{\mathcal{A}}$ by

$$
\mathrm{B}(C, C):=1 \text {, and } \mathrm{B}(C, D):=\prod_{H \in \mathcal{H}(C, D)} a_{H} \text { if } C \neq D .
$$

The Varchenko determinant of the hyperplane arrangement $\mathcal{A}$ is the determinant

$$
\operatorname{det} \mathcal{A}:=\operatorname{det}(\mathrm{B}(C, D))_{C, D \in \mathfrak{C}(\mathcal{A})}
$$

of the matrix associated to the bilinear symmetric form B. The formula of this determinant due to Varchenko is [6, (1.1) Theorem]

$$
\operatorname{det} \mathcal{A}=\prod_{E \in L(\mathcal{A})}\left(1-\mathrm{a}(E)^{2}\right)^{l(E)} .
$$

It is, however, not feasible to directly use this formula to compute a determinant from a certain complexity level. For example, one can not deduce $\operatorname{det} \mathcal{B}_{24}$ directly from it. In this article, we show that we can work around this difficulty for certain hyperplane arrangements if we know the edges which are relevant. In this purpose, we use a clearer definition of the multiplicity $l(E)$ written in an article of Denham and Hanlon [1, 2. The Nullspace of the $B$ Matrices]: First choose a hyperplane $H$ containing $E$. Then $l(E)$ is half the number of chambers $C$ which have the property that $E$ is the minimal edge containing $\bar{C} \cap H$.

We determine the relevant edges in the next section. Then, we compute the Varchenko determinants of some hyperplane arrangements in the last section.

\section{The Relevant Edges}

In this section, we remove in the Varchenko determinant the factors $\left(1-\mathrm{a}(E)^{2}\right)^{l(E)}$ such that $l(E)=0$. In the edge set $L\left(\mathcal{B}_{7}\right)$, for example, we do not need to consider the edges $\left\{x \in \mathbb{R}^{7} \mid x_{1}=x_{2}, x_{4}=x_{5}\right\}$ and $\left\{x \in \mathbb{R}^{7} \mid x_{2}=x_{4}=x_{5}, x_{1}=x_{7}\right\}$ whose multiplicity is 0 . This removal simplifies the computation of the Varchenko determinant.

Take a hyperplane arrangement $\mathcal{A}$ in $\mathbb{R}^{n}$. We say that an edge $E$ of $\mathcal{A}$ is relevant if $l(E) \neq 0$. Denote the relevant edge set of $\mathcal{A}$ by

$$
\mathfrak{R}_{\mathcal{A}}:=\left\{E \in L_{\mathcal{A}} \mid l(E) \neq 0\right\} .
$$

To determine $\mathfrak{R}_{\mathcal{A}}$, we have to consider the faces of the chambers. Recall that the face set of a chamber $C$ in $\mathfrak{C}_{\mathcal{A}}$ resp. of the chambers 
in $\mathfrak{C}_{\mathcal{A}}$ is

$\mathcal{F}(C):=\left\{\bar{C} \cap E \mid E \in L_{\mathcal{A}}, \bar{C} \cap E \neq \emptyset\right\} \quad$ resp. $\quad \mathcal{F}\left(\mathfrak{C}_{\mathcal{A}}\right):=\bigcup_{C \in \mathfrak{C}_{\mathcal{A}}} \mathcal{F}(C)$.

Define the following subset of $\mathcal{F}(C)$ resp. $\mathcal{F}\left(\mathfrak{C}_{\mathcal{A}}\right)$

$\mathcal{S}(C):=\{\bar{C} \cap H \mid H \in \mathcal{A}, \bar{C} \cap H \neq \emptyset\} \quad$ resp. $\quad \mathcal{S}\left(\mathfrak{C}_{\mathcal{A}}\right):=\bigcup_{C \in \mathfrak{C}_{\mathcal{A}}} \mathcal{S}(C)$.

Lemma 2.1. Let $\mathcal{A}$ be a hyperplane arrangement in $\mathbb{R}^{n}$. Then

$$
\mathfrak{R}_{\mathcal{A}}=\left\{\langle F\rangle \mid F \in \mathcal{S}\left(\mathfrak{C}_{\mathcal{A}}\right)\right\} .
$$

Proof. Let $F \in \mathcal{S}\left(\mathfrak{C}_{\mathcal{A}}\right)$. We have,

$$
\langle F\rangle=\bigcap_{\substack{H \in \mathcal{A} \\ F \subseteq H}} H .
$$

By the definition, there exist a chamber $C$ and a hyperplane $H$ such that $\bar{C} \cap H=F$. Since $\langle F\rangle$ is the minimal edge containing $F$, then $l(\langle F\rangle) \geq 1$.

Now, take $E \in L_{\mathcal{A}} \backslash\left\{\langle F\rangle \mid F \in \mathcal{S}\left(\mathfrak{C}_{\mathcal{A}}\right)\right\}$. Suppose that there exist a chamber $C$ and a hyperplane $H$ such that $E$ is the minimal edge containing $F=\bar{C} \cap H$. It means that $E \varsubsetneqq\langle F\rangle$, which impossible since $F \subseteq E$.

Remark that, in the formula $l(E)=n(E) p(E)$, we always have $n(E) \geq 1$. So only the factor $p(E)$ could be considered for deciding which edges are relevant.

Proposition 2.2. Let $\mathcal{A}$ be a hyperplane arrangement in $\mathbb{R}^{n}$. For every relevant edge $E$, we fix a hyperplane $H_{E}$ of $\mathcal{A}$ containing it. Then,

$$
\begin{aligned}
& \operatorname{det} \mathcal{A}=\prod_{E \in \mathfrak{R}_{\mathcal{A}}}\left(1-\mathrm{a}(E)^{2}\right)^{l(E)} \\
& \text { with } l(E)=\frac{1}{2}\left|\left\{C \in \mathfrak{C}_{\mathcal{A}} \mid\left\langle\bar{C} \cap H_{E}\right\rangle=E\right\}\right| .
\end{aligned}
$$

Proof. It is clear that

$$
\prod_{E \in L_{\mathcal{A}}}\left(1-\mathrm{a}(E)^{2}\right)^{l(E)}=\prod_{E \in \Re_{\mathcal{A}}}\left(1-\mathrm{a}(E)^{2}\right)^{l(E)} .
$$

Recall that $l(E)$ is half the number of chambers $C$ which have the property that $E$ is the minimal edge containing $\bar{C} \cap H_{E}$. From 
Lemma 2.1, we deduce that $E$ is the minimal edge containing $\bar{C} \cap H_{E}$ if and only if $\left\langle\bar{C} \cap H_{E}\right\rangle=E$.

\section{Some Varchenko Determinants}

We compute some Varchenko determinants in this section. Furthermore, the Varchenko determinants of the braid arrangement, and of the hyperplane arrangement associated to the hyperoctahedral group were also computed by Pfeiffer, and Randriamaro [4, 6 Computing the determinants of finite Coxeter groups], but by using tools from group theory.

Central 2-Dimensional Arrangement. This is a hyperplane arrangement $\mathcal{A}=\left\{H_{1}, \ldots, H_{m}\right\}$ in $\mathbb{R}^{2}$ such that the intersection $\bigcap_{i=1}^{m} H_{i}$ is the origin $\{0\}$. An example is the hyperplane arrangement associated to the dihedral group $D_{m}$ having $2 m$ elements with

$$
H_{i}=\left\{x \in \mathbb{R}^{2} \mid x_{1} \cos \frac{(i-1) \pi}{m}+x_{2} \sin \frac{(i-1) \pi}{m}=0\right\} .
$$

Assign the weight $a_{i}$ to the hyperplane $H_{i}$. Then, $\mathfrak{R}_{\mathcal{A}}=\left\{H_{1}, \ldots, H_{m},\{0\}\right\}$, and

- $\mathrm{a}\left(H_{i}\right)=a_{i}$ with $l\left(H_{i}\right)=2$,

- $\mathrm{a}(\{0\})=\prod_{i=1}^{m} a_{i}$ with $l(\{0\})=m-2$.

Then,

$$
\operatorname{det} \mathcal{A}=\left(1-\prod_{i=1}^{m} a_{i}^{2}\right)^{m-2} \prod_{j=1}^{m}\left(1-a_{j}^{2}\right)^{2} .
$$

General Position and the Hypercubic Arrangement. A hyperplane arrangement $\mathcal{G}_{n}=\left\{H_{1}, \ldots, H_{n+1}\right\}$ in $\mathbb{R}^{n}$ is in general position if, for every subset $P$ of $[n]$ such that $|P|=p$, we have $\operatorname{dim} \bigcap_{i \in P} H_{i}=n-p$. This is the case for the hyperplane arrangement such that

- $\forall i \in[n], H_{i}=\left\{x \in \mathbb{R}^{n} \mid x_{i}=0\right\}$,

- $H_{n+1}=\left\{x \in \mathbb{R}^{n} \mid x_{1}+\cdots+x_{n}=1\right\}$.

Assign the weight $a_{i}$ to the hyperplane $H_{i}$.

And for $\alpha, \beta$ in $\mathbb{R}$ with $\alpha \neq \beta$, the hypercubic arrangement is the hyperplane arrangement $\mathcal{C}_{n}=\left\{H_{1, \alpha}, H_{1, \beta}, \ldots, H_{n, \alpha}, H_{n, \beta}\right\}$ in $\mathbb{R}^{n}$ such that $H_{i, \alpha \text { resp. } \beta}=\left\{x \in \mathbb{R}^{n} \mid x_{i}=\alpha\right.$ resp. $\left.\beta\right\}$.

Assign the weight $a_{i, \alpha}$ resp. $a_{i, \beta}$ to the hyperplane $H_{i, \alpha}$ resp. $H_{i, \beta}$.

Both hyperplane arrangements have the property

$\forall H \in \mathcal{G}_{n}$ resp. $\mathcal{C}_{n}, \forall C \in \mathfrak{C}\left(\mathcal{G}_{n}\right)$ resp. $\mathfrak{C}\left(\mathcal{C}_{n}\right),\langle C \cap H\rangle=H$ or $\langle C \cap H\rangle=\emptyset$. 
From Lemma 2.1, we deduce that $\mathfrak{R}\left(\mathcal{G}_{n}\right)=\mathcal{G}_{n}$, and $\mathfrak{R}\left(\mathcal{C}_{n}\right)=\mathcal{C}_{n}$. Moreover, since $\left|\mathcal{G}_{n}\right|=2^{n}-1$, and $\left|\mathcal{C}_{n}\right|=3^{n}$, then

$$
\begin{gathered}
\left|\left\{C \in \mathcal{G}_{n} \mid H \in \mathcal{G}_{n},\langle C \cap H\rangle=H\right\}\right|=2^{n}-2 \\
\text { and }\left|\left\{C \in \mathcal{C}_{n} \mid H \in \mathcal{C}_{n},\langle C \cap H\rangle=H\right\}\right|=2 \times 3^{n-1} .
\end{gathered}
$$

Thus

$\operatorname{det} \mathcal{G}_{n}=\prod_{i=1}^{n}\left(1-a_{i}^{2}\right)^{2^{n-1}-1}$ and $\operatorname{det} \mathcal{C}_{n}=\prod_{i=1}^{n}\left(1-a_{i, \alpha}^{2}\right)^{3^{n-1}}\left(1-a_{i, \beta}^{2}\right)^{3^{n-1}}$

Braid Arrangement. This consists of the $\left(\begin{array}{l}n \\ 2\end{array}\right)$ hyperplanes $H_{i, j}=\left\{x \in \mathbb{R}^{n} \mid x_{i}-x_{j}=0\right\}$, with $1 \leq i<j \leq n$. We assign the weight $a_{i, j}$ to the hyperplane $H_{i, j}$.

Proposition 3.1. Let $n \geq 2$. We have

$$
\operatorname{det} \mathcal{B}_{n}=\prod_{\substack{\left.I \in\right|^{[n]} \\
|I| \geq 2}}\left(1-\prod_{\{i, j\} \in\left(\begin{array}{l}
I \\
2
\end{array}\right)} a_{i, j}^{2}\right)^{(|I|-2) !(n-|I|+1) !} .
$$

This determinant was also calculated by Duchamp et al. [2, 6.4.2 A Decomposition of $B_{n}$ ] using the diagonal solutions of the YangBaxter equation.

Each chamber of $\mathcal{B}_{n}$ is defined by $\left\{x \in \mathbb{R}^{n} \mid x_{\sigma(1)}>x_{\sigma(2)}>\cdots>\right.$ $\left.x_{\sigma(n)}\right\}$, where $\sigma$ is a permutation of $[n]$. We write $\left\{x_{\sigma(1)}>x_{\sigma(2)}>\right.$ $\left.\cdots>x_{\sigma(n)}\right\}$ for simplicity.

Let $I=\left\{i_{1}, \ldots, i_{r}\right\}$ be a subset of $[n]$, with $|I| \geq 2$. Denote by $E(I)$ the edge

$$
E(I):=\bigcap_{\{i, j\} \in\left(\left\{i_{1}, \ldots, i_{2}\right\}\right.} H_{i, j}
$$

Lemma 3.2. Let $n \geq 2$. We have

$$
\mathfrak{R}\left(\mathcal{B}_{n}\right)=\{E(I)|I \subseteq[n],| I \mid \geq 2\} .
$$

Proof. Consider a hyperplane $H_{s, t}$ of $\mathcal{B}_{n}$, and a permutation $\sigma$ of $[n]$ such that $\sigma(i)=s$ and $\sigma(j)=t$ with $i<j$. Then,

$$
\begin{aligned}
\left\langle H_{s, t} \cap\right. & \left.\overline{\left\{x_{\sigma(1)}>x_{\sigma(2)}>\cdots>x_{\sigma(n)}\right\}}\right\rangle \\
& =\left\langle\left\{x_{\sigma(1)}>\cdots>x_{\sigma(i)}=\cdots=x_{\sigma(j)}>\cdots>x_{\sigma(n)}\right\}\right\rangle \\
& =\left\{x \in \mathbb{R}^{n} \mid x_{\sigma(i)}=x_{\sigma(i+1)}=\cdots=x_{\sigma(j)}\right\} \\
& =E(\{\sigma(i), \sigma(i+1), \ldots, \sigma(j)\}) .
\end{aligned}
$$


Hence, $E(\{\sigma(i), \sigma(i+1), \ldots, \sigma(j)\})$ is the minimal edge containing the face $H_{i_{s}, i_{t}} \cap \overline{\left\{x_{\sigma(1)}>x_{\sigma(2)}>\cdots>x_{\sigma(n)}\right\}}$.

Lemma 3.3. Let $I \subseteq[n]$. Then, $l(E(I))=(|I|-2) !(n-|I|+1)$ !.

Proof. Let $H_{i_{1}, i_{r}}$ be a hyperplane containing $E(I)$. We have to count the chambers $\left\{x_{\sigma(1)}>x_{\sigma(2)}>\cdots>x_{\sigma(n)}\right\}$ such that

$$
\left\langle H_{i_{1}, i_{r}} \cap \overline{\left\{x_{\sigma(1)}>x_{\sigma(2)}>\cdots>x_{\sigma(n)}\right\}}\right\rangle=E(I) .
$$

Let $\nu$ be a permutation of $\{2, \ldots, r-1\}$. These chambers correspond to the chambers having the forms

$$
\begin{aligned}
& \left\{\cdots>x_{i_{1}}>x_{i_{\nu(2)}}>\cdots>x_{i_{\nu(r-1)}}>x_{i_{r}}>\ldots\right\} \\
& \text { and }\left\{\cdots>x_{i_{r}}>x_{i_{\nu(2)}}>\cdots>x_{i_{\nu(r-1)}}>x_{i_{1}}>\ldots\right\} .
\end{aligned}
$$

Because of the coefficient $\frac{1}{2}$ in the multiplicity, we just need to consider the chambers

$$
\begin{aligned}
& \left\{x_{\sigma(1)}>x_{\sigma(2)}>\cdots>x_{\sigma(n)}\right\} \\
& =\left\{\cdots>x_{i_{1}}>x_{i_{\nu(2)}}>\cdots>x_{i_{\nu(r-1)}}>x_{i_{r}}>\ldots\right\} .
\end{aligned}
$$

Let $i \in[n]$ such that $\sigma(i)=i_{1}$. We have:

- $(n-r)$ ! possibilities for the sequence $(\sigma(1), \ldots, \sigma(i-1), \sigma(i+r), \ldots, \sigma(n))$,

- $(r-2)$ ! possibilities for the sequence $(\sigma(i+1), \ldots, \sigma(i+r-2))$,

- and $n-r+1$ possibilities to choose $i$ since we must have $i \in[n-r+1]$.

Then $l(E(I))=(n-r) ! \times(r-2) ! \times(n-r+1)=(r-2) !(n-r+1)$ !.

We obtain Proposition 3.1 by combining Proposition 2.2 and Lemma 3.3.

Hyperplane Arrangement Associated to Hyperoctahedral Group. Let $[ \pm n]:=\{-n, \ldots,-2,-1,1,2, \ldots, n\}$. Denote $\overline{2^{[ \pm n]}}$ the subset of $2^{[ \pm n]}$ having the following properties:

- the elements of $\overline{2^{[ \pm n]}}$ are the elements $\left\{i_{1}, \ldots, i_{t}\right\}$ of $2^{[ \pm n]}$ such that $\left|i_{r}\right| \neq\left|i_{s}\right|$ if $r \neq s$

- and if $\left\{i_{1}, \ldots, i_{t}\right\} \in \overline{2^{[ \pm n]}}$, then $\left\{-i_{1}, \ldots,-i_{t}\right\} \notin \overline{2^{[ \pm n]}}$. 
For example,

$$
\begin{aligned}
\overline{2^{[ \pm 3]}}= & \{\emptyset,\{1\},\{2\},\{3\}, \\
& \{1,2\},\{-1,2\},\{1,3\},\{-1,3\},\{2,3\},\{-2,3\}, \\
& \{1,2,3\},\{-1,2,3\},\{-1,-2,3\},\{1,-2,3\}\} .
\end{aligned}
$$

The hyperplane arrangement $\mathcal{O}_{n}$ associated to the hyperoctahedral group $B_{n}$ consists of

- the $\left(\begin{array}{l}n \\ 2\end{array}\right)$ hyperplanes $H_{i, j}=\left\{x \in \mathbb{R}^{n} \mid x_{i}-x_{j}=0\right\}$ with $1 \leq i<j \leq n$,

- the $\left(\begin{array}{l}n \\ 2\end{array}\right)$ hyperplanes $H_{-i, j}=\left\{x \in \mathbb{R}^{n} \mid x_{i}+x_{j}=0\right\}$ with $1 \leq i<j \leq n$,

- the $n$ hyperplanes $H_{i}=\left\{x \in \mathbb{R}^{n} \mid x_{i}=0\right\}$ with $i \in[n]$.

We assign the weights $a_{i, j}$ to the hyperplanes $H_{i, j}$, the weights $a_{-i, j}$ to the hyperplanes $H_{-i, j}$, and the weights $a_{i}$ to the hyperplanes $H_{i}$.

Proposition 3.4. Let $n \geq 2$. We have

$$
\begin{aligned}
\operatorname{det} \mathcal{O}_{n}= & \prod_{\substack{J \in 2[ \pm n] \\
|J| \geq 2}}\left(1-\prod_{\{i, j\} \in\left(\begin{array}{l}
J \\
2
\end{array}\right)} a_{i, j}^{2}\right)^{2^{n-|J|+1}(|J|-2) !(n-|J|+1) !} \\
& \prod_{\substack{I \in 2^{[n]} \\
|I| \geq 1}}\left(1-\prod_{i \in I} a_{i}^{2} \prod_{\{i, j\} \in\left(\begin{array}{l}
I \\
2
\end{array}\right)} a_{i, j}^{2} a_{-i, j}^{2}\right)^{2^{n-1}(|I|-1) !(n-|I|) !} .
\end{aligned}
$$

Each chamber of $\mathcal{O}_{n}$ is defined by

$$
\left\{x \in \mathbb{R}^{n} \mid \epsilon_{1} x_{\sigma(1)}>\epsilon_{2} x_{\sigma(2)}>\cdots>\epsilon_{n} x_{\sigma(n)}>0\right\},
$$

where $\epsilon_{i} \in[ \pm 1]$ and $\sigma$ is a permutation of $[n]$.

For $J=\left\{\epsilon_{1} i_{1}, \epsilon_{2} i_{2}, \ldots, \epsilon_{r} i_{r}\right\} \in \overline{2^{[ \pm n]}}$, with $|J| \geq 2$, denote $E(J)$ the edge

$$
E(J):=\left\{x \in \mathbb{R}^{n} \mid \epsilon_{1} x_{i_{1}}=\epsilon_{2} x_{i_{2}}=\cdots=\epsilon_{r} x_{i_{r}}\right\} .
$$

And for $I=\left\{i_{1}, i_{2}, \ldots, i_{r}\right\} \subseteq 2^{[n]}$, with $r \geq 1$, denote $E\left(I_{0}\right)$ the edge

$$
E\left(I_{0}\right):=\left\{x \in \mathbb{R}^{n} \mid x_{i_{1}}=x_{i_{2}}=\cdots=x_{i_{r}}=0\right\} .
$$

Lemma 3.5. Let $n \geq 2$. We have

$$
\mathfrak{R}\left(\mathcal{O}_{n}\right)=\left\{E(J)\left|J \in \overline{2^{[ \pm n]}},\right| J \mid \geq 2\right\} \cup\left\{E\left(I_{0}\right)\left|I \in 2^{[n]},\right| I \mid \geq 1\right\} .
$$

Proof. Consider a hyperplane $H_{\epsilon_{s} s, \epsilon_{t} t}$ of $\mathcal{O}_{n}$, and a permutation $\sigma$ of $[n]$ such that $\sigma(i)=s$ and $\sigma(j)=t$ with $i<j$. If 
- $\left\{\epsilon_{s} s, \epsilon_{t} t\right\}=\left\{\epsilon_{i} \sigma(i), \epsilon_{j} \sigma(j)\right\}$, then

$$
\begin{aligned}
\langle & \left.H_{\epsilon_{s} s, \epsilon_{t}} \cap \overline{\left\{\epsilon_{1} x_{\sigma(1)}>\epsilon_{2} x_{\sigma(2)}>\cdots>\epsilon_{n} x_{\sigma(n)}>0\right\}}\right\rangle \\
= & \left\langle\left\{\epsilon_{1} x_{\sigma(1)}>\cdots>\epsilon_{i} x_{\sigma(i)}=\cdots=\epsilon_{j} x_{\sigma(j)}>\cdots>\epsilon_{n} x_{\sigma(n)}>0\right\}\right\rangle \\
= & \left\{\epsilon_{i} x_{\sigma(i)}=\epsilon_{i+1} x_{\sigma(i+1)}=\cdots=\epsilon_{j} x_{\sigma(j)}\right\} \\
= & E\left(\left\{\epsilon_{i} \sigma(i), \epsilon_{i+1} \sigma(i+1), \ldots, \epsilon_{j} \sigma(j)\right\}\right) . \\
& \bullet\left\{\epsilon_{s} i_{s}, \epsilon_{t} i_{t}\right\} \neq\left\{\epsilon_{i} \sigma(i), \epsilon_{j} \sigma(j)\right\}, \text { then } \\
& \left\langle H_{\epsilon_{s} s, \epsilon_{t}} \cap \overline{\left\{\epsilon_{1} x_{\sigma(1)}>\epsilon_{2} x_{\sigma(2)}>\cdots>\epsilon_{n} x_{\sigma(n)}>0\right\}}\right\rangle \\
& =\left\langle\left\{\epsilon_{1} x_{\sigma(1)}>\cdots>\epsilon_{i-1} x_{\sigma(i-1)}>x_{\sigma(i)}=\cdots=x_{\sigma(n)}=0\right\}\right\rangle \\
& =\left\{x_{\sigma(i)}=x_{\sigma(i+1)}=\cdots=x_{\sigma(n)}=0\right\} \\
& =E(\{0, \sigma(i), \sigma(i+1), \ldots, \sigma(n)\}) .
\end{aligned}
$$

Consider a hyperplane $H_{u}$ of $\mathcal{O}_{n}$, and a permutation $\sigma$ of $[n]$ such that $\sigma(i)=u$. Then,

$$
\begin{aligned}
& \left\langle H_{u} \cap \overline{\left\{\epsilon_{1} x_{\sigma(1)}>\epsilon_{2} x_{\sigma(2)}>\cdots>\epsilon_{n} x_{\sigma(n)}>0\right\}}\right\rangle \\
& =\left\langle\left\{\epsilon_{1} x_{\sigma(1)}>\cdots>\epsilon_{i-1} x_{\sigma(i-1)}>x_{\sigma(i)}=\cdots=x_{\sigma(n)}=0\right\}\right\rangle \\
& =\left\{x_{\sigma(i)}=x_{\sigma(i+1)}=\cdots=x_{\sigma(n)}=0\right\} \\
& =E(\{0, \sigma(i), \sigma(i+1), \ldots, \sigma(n)\}) .
\end{aligned}
$$

Lemma 3.6. Let $E(J), E\left(I_{0}\right) \in \mathfrak{R}\left(O_{n}\right)$. Then,

$$
\begin{aligned}
& \mathrm{a}(E(J))=\prod_{\{s, t\} \in\left(\begin{array}{l}
J \\
2
\end{array}\right)} a_{s, t} \\
& \text { with } \quad l(E(J))=2^{n-|J|+1}(|J|-2) !(n-|J|+1) !, \\
& \mathrm{a}\left(E\left(I_{0}\right)\right)=\prod_{u \in I} a_{u} \prod_{\{s, t\} \in\left(\begin{array}{l}
I \\
2
\end{array}\right)} a_{s, t} a_{-s, t} \\
& \text { with } l\left(E\left(I_{0}\right)\right)=2^{n-1}(|I|-1) !(n-|I|) !
\end{aligned}
$$

Proof. We have

$$
\mathrm{a}(E(J))=\prod_{\substack{H \in \mathcal{O}_{n} \\
E(J) \subseteq H}} \mathrm{a}(H)=\prod_{\{s, t\} \in\left(\begin{array}{l}
J \\
2
\end{array}\right)} a_{s, t},
$$


and

$$
\mathrm{a}\left(E\left(I_{0}\right)\right)=\prod_{\substack{H \in \mathcal{O}_{n} \\
E\left(I_{0}\right) \subseteq H}} \mathrm{a}(H)=\prod_{u \in I} a_{u} \prod_{\{s, t\} \in\left(\begin{array}{l}
I \\
2
\end{array}\right)} a_{s, t} a_{-s, t} .
$$

To the edge $E(J)$, assign the hyperplane $H_{\epsilon_{1} i_{1}, \epsilon_{r} i_{r}}$ containing it. We first have to count the chambers $\left\{\epsilon_{1} x_{\sigma(1)}>\epsilon_{2} x_{\sigma(2)}>\cdots>\epsilon_{n} x_{\sigma(n)}>\right.$ $0\}$ such that

$$
\left\langle H_{\epsilon_{1} i_{1}, \epsilon_{r} i_{r}} \cap \overline{\left\{\epsilon_{1} x_{\sigma(1)}>\epsilon_{2} x_{\sigma(2)}>\cdots>\epsilon_{n} x_{\sigma(n)}>0\right\}}\right\rangle=E(J) .
$$

Let $\nu$ be a permutation of $\{2, \ldots, r-1\}$. These chambers correspond to the chambers having the forms

$$
\begin{aligned}
& \left\{\cdots>\epsilon_{1} x_{i_{1}}>\epsilon_{\nu(2)} x_{i_{\nu(2)}}>\cdots>\epsilon_{\nu(r-1)} x_{i_{\nu(r-1)}}>\epsilon_{r} x_{i_{r}}>\ldots\right\}, \\
& \left\{\cdots>\epsilon_{r} x_{i_{r}}>\epsilon_{\nu(2)} x_{i_{\nu(2)}}>\cdots>\epsilon_{\nu(r-1)} x_{i_{\nu(r-1)}}>\epsilon_{1} x_{i_{1}}>\ldots\right\}, \\
& \left\{\cdots>-\epsilon_{1} x_{i_{1}}>\epsilon_{\nu(2)} x_{i_{\nu(2)}}>\cdots>\epsilon_{\nu(r-1)} x_{i_{\nu(r-1)}}>-\epsilon_{r} x_{i_{r}}>\ldots\right\}, \\
& \text { and } \\
& \left\{\cdots>-\epsilon_{r} x_{i_{r}}>\epsilon_{\nu(2)} x_{i_{\nu(2)}}>\cdots>\epsilon_{\nu(r-1)} x_{i_{\nu(r-1)}}>-\epsilon_{1} x_{i_{1}}>\ldots\right\} .
\end{aligned}
$$

Because of the coefficient $\frac{1}{2}$ in the multiplicity and the symmetry of the signed permutation, we just need to consider the chambers

$$
\begin{aligned}
& \left\{\epsilon_{1} x_{\sigma(1)}>\epsilon_{2} x_{\sigma(2)}>\cdots>\epsilon_{n} x_{\sigma(n)}>0\right\} \\
& =\left\{\cdots>\epsilon_{1} x_{i_{1}}>\epsilon_{\nu(2)} x_{i_{\nu(2)}}>\cdots>\epsilon_{\nu(r-1)} x_{i_{\nu(r-1)}}>\epsilon_{r} x_{i_{r}}>\ldots\right\}
\end{aligned}
$$

and multiply the obtained cardinality by 2 . Let $i \in[n]$ such that $\sigma(i)=i_{1}$. We have:

- $2^{n-r}(n-r)$ ! possibilities for the sequence

$$
\left(\epsilon_{1} \sigma(1), \ldots, \epsilon_{i-1} \sigma(i-1), \epsilon_{i+r} \sigma(i+r), \ldots, \epsilon_{n} \sigma(n)\right) \text {, }
$$

- $(r-2)$ ! possibilities for the sequence

$$
\left(\epsilon_{i+1} \sigma(i+1), \ldots, \epsilon_{i+r-2} \sigma(i+r-2)\right) \text {, }
$$

- and $n-r+1$ possibilities to choose $i$ since we must have $i \in[n-r+1]$.

Then

$$
\begin{aligned}
l(E(J)) & =2 \times 2^{n-r}(n-r) ! \times(r-2) ! \times(n-r+1) \\
& =2^{n-r+1}(r-2) !(n-r+1) ! .
\end{aligned}
$$

To the edge $E\left(I_{0}\right)$, assign the hyperplane $H_{i_{r}}$ containing it. Now, we have to count the chambers $\left\{\epsilon_{1} x_{\sigma(1)}>\epsilon_{2} x_{\sigma(2)}>\cdots>\epsilon_{n} x_{\sigma(n)}>0\right\}$ such that

$$
\left\langle H_{i_{r}} \cap \overline{\left\{\epsilon_{1} x_{\sigma(1)}>\epsilon_{2} x_{\sigma(2)}>\cdots>\epsilon_{n} x_{\sigma(n)}>0\right\}}\right\rangle=E\left(I_{0}\right) .
$$


Let $\nu$ be a permutation of $[r-1]$. Those chambers correspond to the chambers having the forms

$\begin{array}{ll} & \left\{\cdots>x_{i_{r}}>\epsilon_{\nu(r-1)} x_{i_{\nu(r-1)}}>\cdots>\epsilon_{\nu(2)} x_{i_{\nu(2)}}>\epsilon_{\nu(1)} x_{i_{\nu(1)}}>0\right\} \\ \text { and } \quad & \left\{\cdots>-x_{i_{r}}>\epsilon_{\nu(r-1)} x_{i_{\nu(r-1)}}>\cdots>\epsilon_{\nu(2)} x_{i_{\nu(2)}}>\epsilon_{\nu(1)} x_{i_{\nu(1)}}>0\right\} .\end{array}$

Because of the coefficient $\frac{1}{2}$ in the multiplicity, we just need to consider the chambers

$$
\begin{aligned}
& \left\{\epsilon_{1} x_{\sigma(1)}>\epsilon_{2} x_{\sigma(2)}>\cdots>\epsilon_{n} x_{\sigma(n)}>0\right\} \\
& =\left\{\cdots>x_{i_{r}}>\epsilon_{\nu(r-1)} x_{i_{\nu(r-1)}}>\cdots>\epsilon_{\nu(2)} x_{i_{\nu(2)}}>\epsilon_{\nu(1)} x_{i_{\nu(1)}}>0\right\} .
\end{aligned}
$$

We have:

- $2^{n-r}(n-r)$ ! possibilities for the sequence $\left(\epsilon_{1} \sigma(1), \ldots, \epsilon_{n-r} \sigma(n-r)\right)$,

- and $2^{r-1}(r-1)$ ! possibilities for the sequence $\left(\left(\epsilon_{n-r+2} \sigma(n-r+2), \ldots, \epsilon_{n} \sigma(n)\right)\right.$.

Then $l\left(E\left(I_{0}\right)\right)=2^{n-r}(n-r) ! \times 2^{r-1}(r-1) !=2^{n-1}(r-1) !(n-r) !$.

We obtain Proposition 3.4 by combining Proposition 2.2 and Lemma 3.6.

\section{REFERENCES}

[1] G. Denham, P. Hanlon: Some Algebraic Properties of the SchechtmanVarchenko Bilinear Form, MSRI Publications (38) (1999), 149-176.

[2] G. Duchamp, A. Klyachko, D. Krob, J.-Y. Thibon: Noncommutative Symmetric Functions III: Deformations of Cauchy and Convolution Algebras, Discrete Math. Theor. Comput. Sci. (1) (1997), 159-216.

[3] P. Orlik, H. Terao: Arrangements of Hyperplanes, Grundlehren der Mathematischen Wissenschaften, Springer, 1992.

[4] G. Pfeiffer, H. Randriamaro: The Varchenko Determinant of a Coxeter Arrangement, J. Group Theory (21) 4 (2018), 651-665.

[5] A. Varchenko: The Euler Beta-function, the Vandermonde Determinant, Legendre's Equation, and Critical Values of Linear Functions on a Configuration of Hyperplanes I, Math. USSR Izv. (35) 3 (1990).

[6] A. Varchenko: Bilinear Form of Real Configuration of Hyperplanes, Adv. Math. (97) 1 (1993), 110-144.

Hery Randriamaro was visiting fellow at International Centre for Theoretical Physics and Leibniz fellow at Mathematisches Forschungsinstitut Oberwolfach. 
His research interests revolve around Varchenko determinant, Tutte polynomial, and Solomon algebra. All these topics are connected to hyperplane arrangements.

(Hery Randriamaro) Mathematisches Forschungsinstitut OberwolFach, Schwarzwaldstrasse 9-11, 77709 Oberwolfach, Germany

E-mail address: hery.randriamaro@outlook.com 